\title{
Téoros
}

Revue de recherche en tourisme

\section{Perception du Canada touristique par les Américains}

\section{L'Enquête Longwoods II}

\section{Pierre Bellerose}

Volume 9, numéro 2, juillet 1990

Le marché américain

URI : https://id.erudit.org/iderudit/1080030ar

DOI : https://doi.org/10.7202/1080030ar

Aller au sommaire du numéro

Éditeur(s)

Université du Québec à Montréal

ISSN

0712-8657 (imprimé)

1923-2705 (numérique)

Découvrir la revue

Citer cet article

Bellerose, P. (1990). Perception du Canada touristique par les Américains :

l'Enquête Longwoods II. Téoros, 9(2), 31-35. https://doi.org/10.7202/1080030ar d'utilisation que vous pouvez consulter en ligne.

https://apropos.erudit.org/fr/usagers/politique-dutilisation/ 


\section{Perception du Canada touristique par les Américains \\ L'Enquête Longwoods II}

Pierre Bellerose*

Aussi paradoxal que cela puisse paraître, il y a quelques annees à peine, on connaissait très peu de choses du marché américain hormis les questions de volumes (et encore là!). Ainsi, par exemple, la publicité du Canada aux États-Unis dans les annees '70 et au début des années ' 80 , mettait l'accent principalement sur le plein air, les grands espaces et la nature sans que l'on puisse vérifier la perception de cette image auprès des Américains.

L'enquête de la firme Longwoods en 1985 , auprès de 9000 Américains, a permis de découvrir qu'une majorité d'Américains cherchaient beaucoup plus que cela et qu'il fallait réajuster notre approche. L'enquête Longwoods de 1985 a servi de base a la campagne de publicité: "Le Canada: le Monde à votre porte" (The World Next Door), qui montre le Canada comme une destination étrangère et accueillante en mettant davantage $1^{t}$ accent sur les differences culturelles et le tourisme urbain.

Pour permettre, entre autres, de vérifier l'évolution du marché américain et de la perception du Canada depuis 1985, la firme Longwoods aentrepris une seconde edition de l'enquêtte, auprès du marché américain des voyages d'agrément. Cet article brossera un tableau des résultats préliminaires de cette enquête en ce qui concerne la perception du Canada par les Américains.

\section{La méthodologie}

La firme Longwoods a fait parvenir, par l'entremise de la compagnie américaine NFO Research Inc., le même questionnaire qu'en 1985 a 24000 foyers américains d'octobre 1989 à février 1990 . L'objectif était le même qu'en 1985 , soit de connaître

Pierre Bellerose eat directeur de la recherche of du développement a l'Office des congrés ot du touriame du Grand Montrésl. les habitudes des voyages d'agrément des Américains, ce qui exclut toutes les formes de voyages d'affaires (congrès, foires commerciales, affaires, voyages-motivation). La distribution des questionnaires a été faite de façon à couvrir toutes les régions caractéristiques des États-Unis.

18400 questionnaires furent complétés pour un taux de réponses élevé de $67 \%$. Ce nombre considérable de questionnaires complétés permettra, lorsque les données seront disponibles, une analyse beaucoup plus segmentce que dans cet article. Cette étude a té réalisée au coût de 2 millions de dollars U.S.+

\section{Les résultats}

\section{Le Canada}

Globalement, les données de l'enquête Longwoods ' 89 montre que les réajustements des campagnes canadiennes depuis trois (3) ans ont porté fruit sur le marché des voyages d'agrément tant en termes de volumes qu'en termes de perception. Ainsi, le tableau 1 nous montre 1'evolution du nombre de voyages d'agrément depuis 1985 au Canada par des Américains, par type de voyage.

En fait, ce tableau nous montre une augmentation pour tous les types de voyages saufen ce qui concerne le plein airet les centres de villégiature. Cela vient confirmer les conclusions de l'étude de 1985 où on constatait que le plein air et les grands espaces n'étaient pas le principal déclencheur pour les Américains. À l'inverse, le tourisme urbain semble être un des grands gagnants de cette étude (après les croisières qui constituent un marché plus marginal et qui touchent surtout la Colombie-Britannique), puisque:

a) le tourisme urbain comme destination principale des Américains au Canada a augmenté de $24 \%$ entre
1985 et 1989 pour atteindre près de 1,5 million de voyages;

b) la composante urbaine est aussi très présente dans le tourisme itinérant et ce dernier segment a augmenté de $22 \%$ entre 1985 et 1989 . Ce type de voyage demeure, et de loin, le plus important avec près de 3 millions de voyages en 1989.

Les grands espaces, les parcs nationaux et les activités de plein air par exemple sont quelque peu délaissés par les Américains au profit de la fébrilité citadine, de la culture et de la différence.

Les tableaux 2 et 3 permettent d'evaluer 1'image du Canada par rapport à celle des États-Unis, en ce qui conceme les deux principaux types de voyages des Américains au Canada: le tourisme itinérant et le tourisme urbain. On remarque des différences de perception non négligeables entre les deux (2) types de voyages. Ainsi, le Canada est globalement perçu beaucoup plus positivement que les États-Unis en ce qui concerne le tourisme itinerant (tableau 2). Cela s'explique surtout parce que les Américains considerent le Canada comme une destination attirante, ayant un cachet étranger et offrant des paysages spectaculaires dans le cadre d'un circuit en automobile d'une dizaine de jours. Historiquement, les campagnes publicitaires du Canada sur ce marché ont mis beaucoup l'emphase sur les paysages spectaculaires qui s'intègrent bien dans le concept du circuit. De plus, ces campagnes ont mise surd'autres aspects que les grands espaces, ce qui donne l'image d'une destination au contenu varie. II n'est donc pas étonnant que le produit "tourisme itinérant" frappe l'imagination des Américains. Cependant, on doit souligner la faible perfomance canadienne en ce qui concerne laperception de la qualité de la restauration et de l'hébergement ainsi que le prix. Plusieurs études récentes ${ }^{(1)}$ ont démontré la perte relative de compétitivité du Canada en 
matière de coût face aux États-Unis, surtout depuis la remontée du dollar canadien au milieu des années '80. Elle s'est reflétée surtout au niveau du prix de l'essence, de la restauration et de $1^{\prime}$ hébergement ${ }^{(2)}$ et, semble-t-il, avec encore plus d'acuite au Québec et à Montréal ${ }^{(3)}$. II sera très difficile de changer cette perception sinon en mettant les faits en perspective et en mettant $l^{\dagger}$ accent sur la qualité de l'expérience.

Par ailleurs, en ce qui concerne le tourisme urbain (tableau 3), la situation comparative est moins enviable. En effet, les Américains considèrent leurs villes plus attirantes tout en étant plus divertissantes et moins chères. Eneffet, encore là, le produit touristique canadienest perçu comme plus dispendieux, ce qui confirme que cela est une perception généralisée aux Êtats-Unis. Toutefois, on considere les citadins canadiens plus courtois tout en habitant des villes ayant des particularismes qui leur sont propres. On doit mentionner que "l'exposition" des Amerricains au produit du tourisme urbain canadien est récente et n'a peut-être pas encore donné les résultats escomptés. Cependant, comme pour la notion de tourisme itinerant, il s'agit d'un concept assez large lorsqu'onl'analyse pour tout le Canada. La prochaine section nous permettrade mieux cemer la perception des Américains en ce qui concerne le Québec.

\section{Le Québec}

Le Québec joue un róle particulier dans la campagne publicitaire du gouvemement canadien puisqu'il symbolise ce que les Américains recherchent de plus en plus: la culture et la différence. Le tourisme américain n'est plus, depuis longtemps, un bloc monolithique. Entre autres, la montée du tourisme des communautés hispaniques, noires et asiatiques a change considérablement le portrait d'ensemble. Le touriste américain est plus ouvert et prêt à vivre des expériences différentes ${ }^{(4)}$.

Onpeut présumerqueles Américains seront deplusen plus réceptifspourune destination comme le Québec. D'ailleurs, la nouvelle orientation marketingde Tourisme Canada, depuis l'enquête Longwoods de 1985 , accorde une plus grande importance à ce volet. Bien sûr, d'autres composantes importantes sont intégrées dans les publicités récentes telles l'héritage

\section{Tableau 1}

Nombre de voyages d'agrément de plus de 24 heures effectués au Canada par des Américains âgés de 18 ans et plus, par type de voyage:1989 par rapport à 1985 (000)

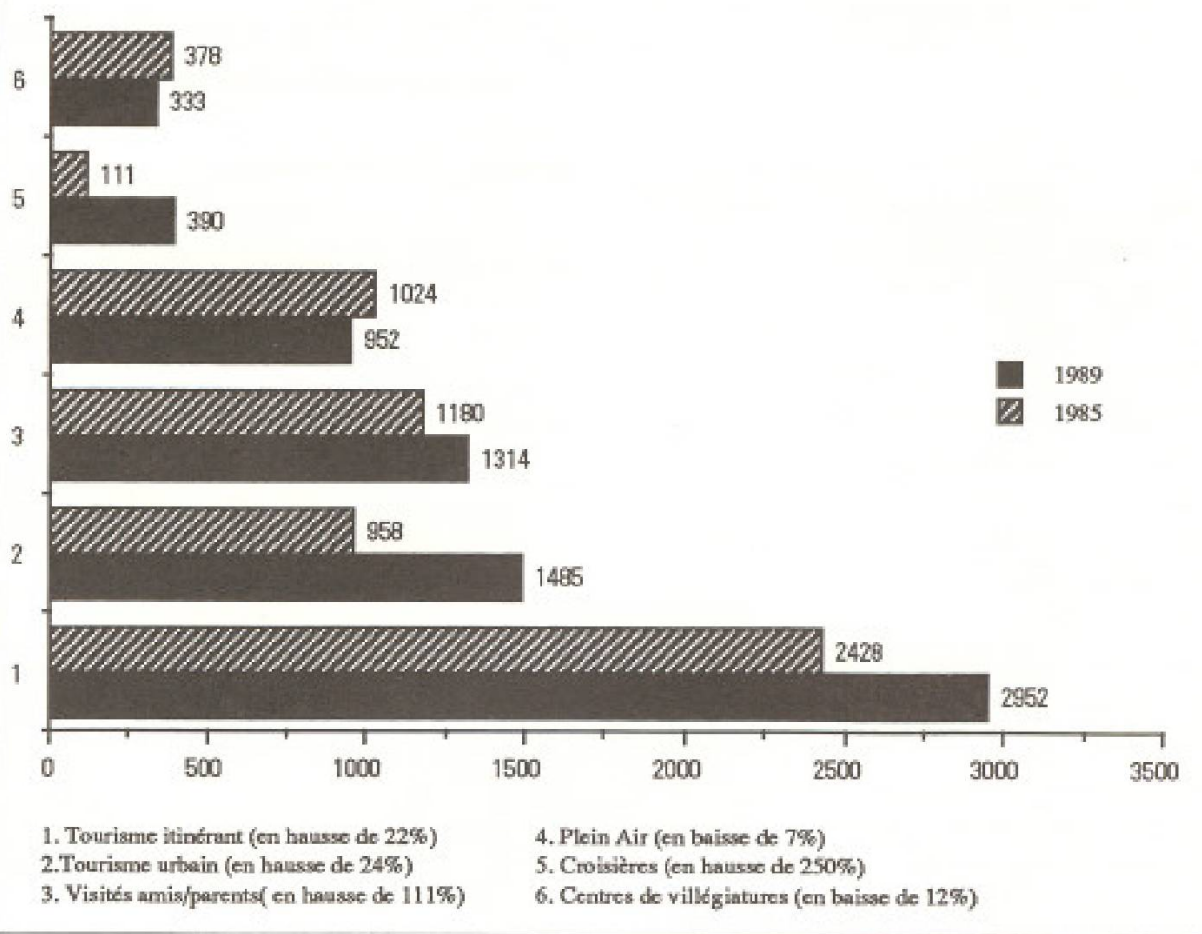

\section{TABLEAU 2}

Image du Canada par rapport à celle des États-Unis en ce qui concerne le tourisme itinérant en 1989

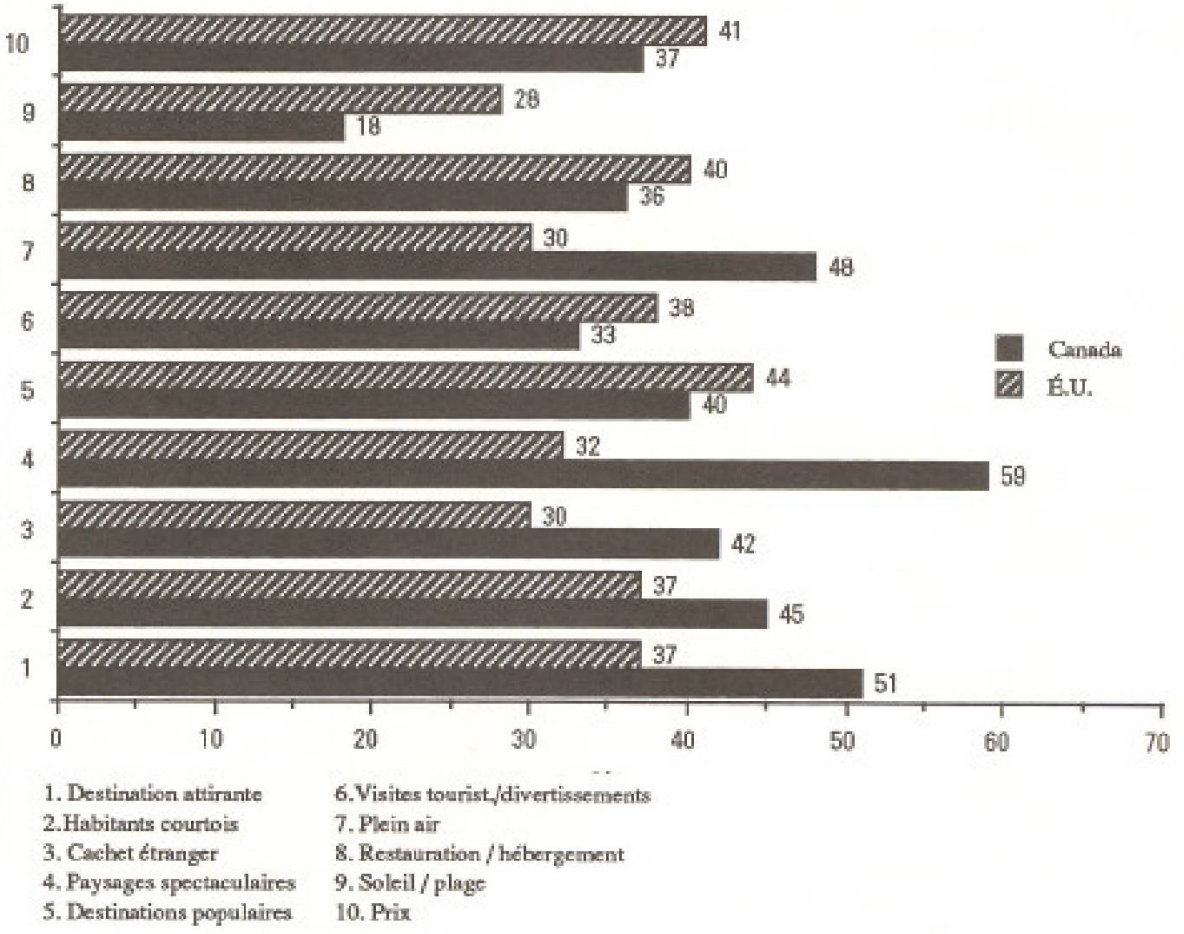


britannique, les autochtones, la présence des groupes ethniques et les grands espaces. Cependant, on ne doit pas oublier que ce sont des éléments que 1"on retrouve aussi aux Êtats-Unismaisàdes niveaux différents. II nous reste maintenant à intensifier nos opérations de promotion pour leur faire partager nos différences.

Le tableau 4 montre que $60 \%$ des Américains interrogés aimeraient beaucoup visiter le Québec. Le Québec se situe au même niveau que les Rocheuses et en deçà de la Cóte du Pacifique qui est la favorite des Américains.

Il est quelque peu surprenant de constater la piètre performance de l'Ontario en comparaison du Québecet des autres régions candiennes surtout lorsqu'on sait que $1^{\prime}$ Ontario est la province canadienne qui dépense le plus en promotion touristique et que la dépense per capita a ce chapitre est plus élevée en Ontario qu ${ }^{7}$ au Québec ${ }^{(s)}$. En effet, malgré cela, seulement $44 \%$ des Américains aimeraient beaucoup visiter 1'Ontario. Deux raisons principales pourraient expliquer cette situation. Historiquement, l'Ontario fut la province canadienne la plus visitée par nos voisins du sud et donc un grand nombre d'entre eux ont déjà expérimenté cette destination. Aussi, et c'est peut-être la principale cause, il semble que $1^{2}$ Ontario ait actuellement beaucoup de difficultés à se positionner aux États-Unis comme une destinationattirante. Malgré les millions dépensés annuellement en promotion, le message passe mal et les Américains considèrent 1'Ontario peu différente des Êtats-Unis. Le Québec, avec moins d'argent, a donc réussi à donner le goût à un grand nombre d'Américains de venir dans la "Belle Province" pour expérimenterun tourisme itinérant différent. Il reste maintenant le plus difficile à faire, c'est-â-dire capitaliser sur cette bonne perception et augmenter le nombre de touristes venant au Québec.

Les résultats illustrés au tableau 5, qui reflètent les destinations urbaines que les Américains trouveraient agréables à visiter, sont encore plus surprenants, Ainsi, les Américains trouveraient beaucoup plus agréable de visiter Montréal $(65 \%)$ que Vancouver ( $53 \%)$, Toronto (50\%) et même New York (42\%). Donc malgré la place importante de New York dans les médias américains, malgré 1'Expo '86 de

\section{TABLEAU 3}

Image du Canada par rapport à celle des États-Unis en ce qui concerne le tourisme urbain en 1989

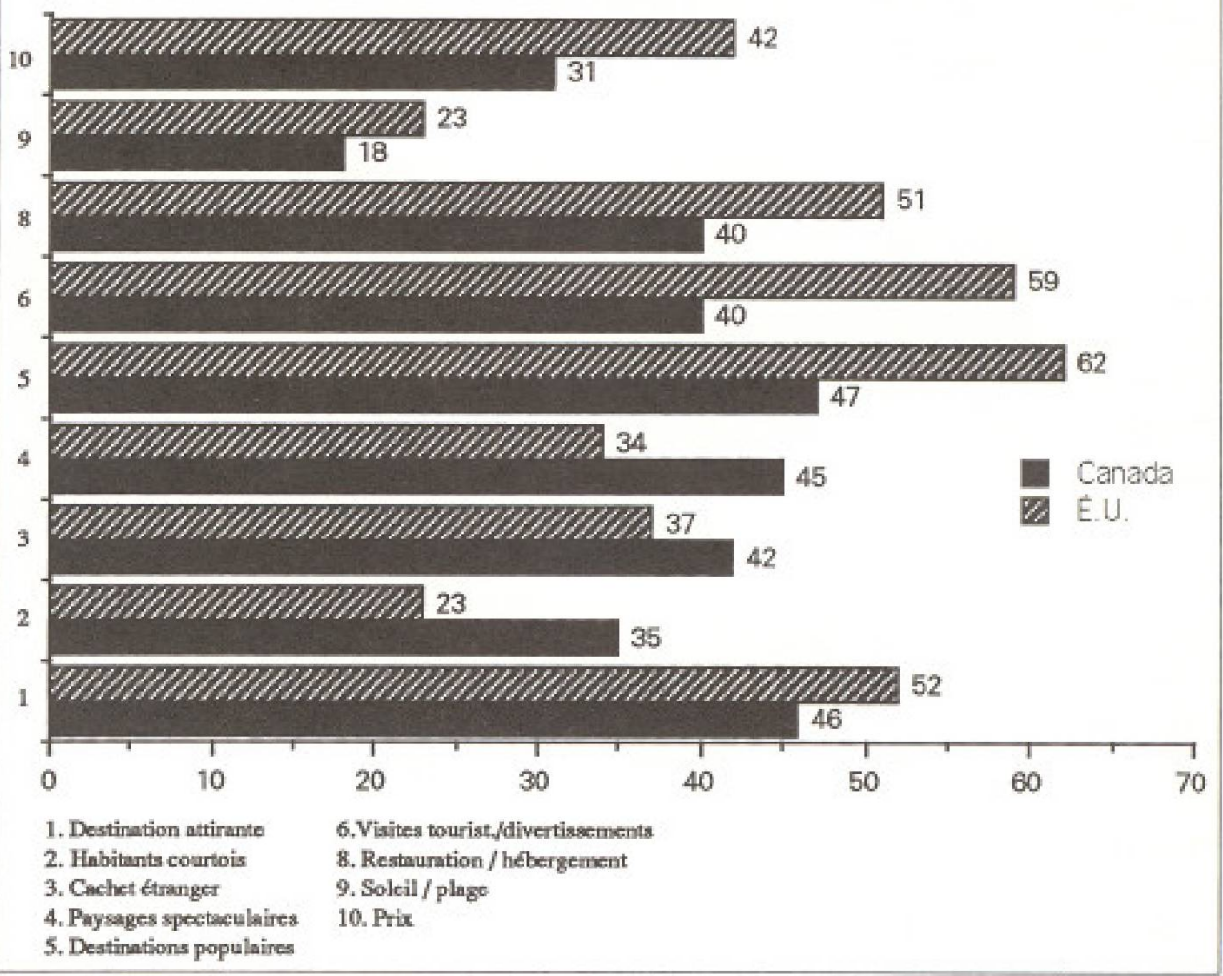

TABLEAU 4

Image des destinations canadiennes de tourisme itinérant

Th de ceux qui sont

enticuranent d'accord

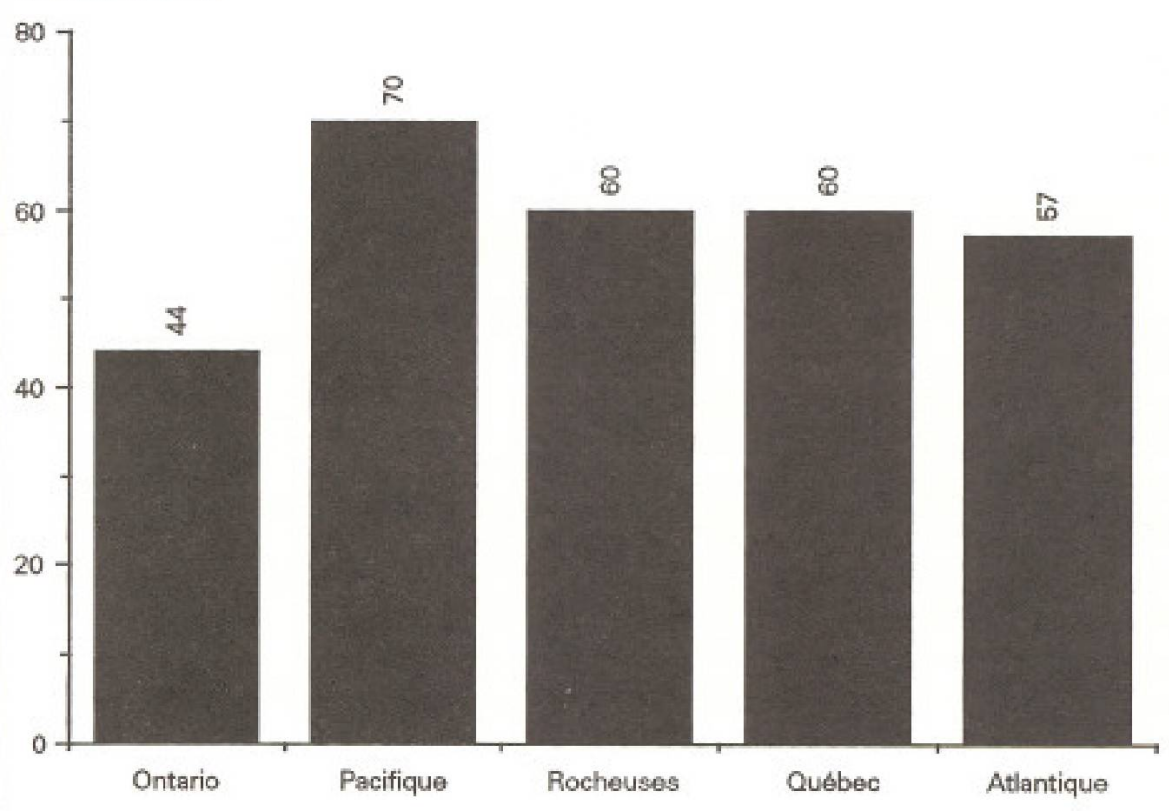

Destination que j'aimerais beaueoup visiter 
Vancouver et les efforts promotionnels de Toronto, Montréal demeure une ville avec une très bonne (et une meilleure) réputation. Cette performance n'est nullement le reflet d'un effort promotionnel soutenu dans les années "80, puisque Montréal a dépensé beaucoup moins d'argent que New York, Toronto et même Vancouver.

On pourrait, en partie, expliquer ce résultat par l'Expo '67 et les Olympiques de 1976 qui ont contribue a faire connaître Montréal à l'étranger comme ville internationale et qui continuent à soutenir (pour combien d'années encore? l' l'image de Montréal. II apparaîtévident que le caractère français de Montréal aide à nous distinguer de nos concurrents. En ce qui concerne les autres villes, New York est perçue comme une ville très peu sécuritaire et Toronto donne l'image d'une ville américaine en plus propre et plus sécuritaire. D'ailleurs, Toronto vit actuellement le même problème que l'Ontario et est en train de réviser complètement son approche promotionnelle aux États-Unis. Toute la question est de savoir si Montréal réussira à profiter dans les prochaines années de cette notoriété qui lui vient en grande partie de son passé et à améliorerl'offre touristique pour renouveler l'intereret. La nouvelle orientation du ministère du Tourisme du Québec et de l'Office des congrès et du tourisme du Grand Montreal (O.C.T.G.M.) $)^{(6)}$ ainsi que la bonification en cours du produit touristique montréalais (agrandissements importantsde plusieurs musées,le Biodôme, investissements majeurs dans le VieuxMontréal et le Vieux-Port, etc.) permettent d'entrevoir l'avenir à moyen terme avec un certain optimisme.

\section{Conclusion}

L'Enquête Longwoods a permis de mesurer l'évolution somme toute positive de l'image du Canada aux États-Unis. Il semble effectivement que le changement d'approche de Tourisme Canada, qui met d'avantage l'accentsur ladiversité culturelle et le caractère urbain, ait porté fruit. Le tableau 6 nous montre en effet que les Américains considèrent maintenant le Canada comme une destination plus agréable et attirante que les États-Unis, alors que c'était l'inverse il y a quatre (4) ans à peine. La situation s'est modifiée du tout au tout entre 1985 et 1989 . En effet, en

\section{TABLEAU 5}

Comparaison entre les villes du Canada et la ville de New York

Th de ceux qui sont

entièrement daceord

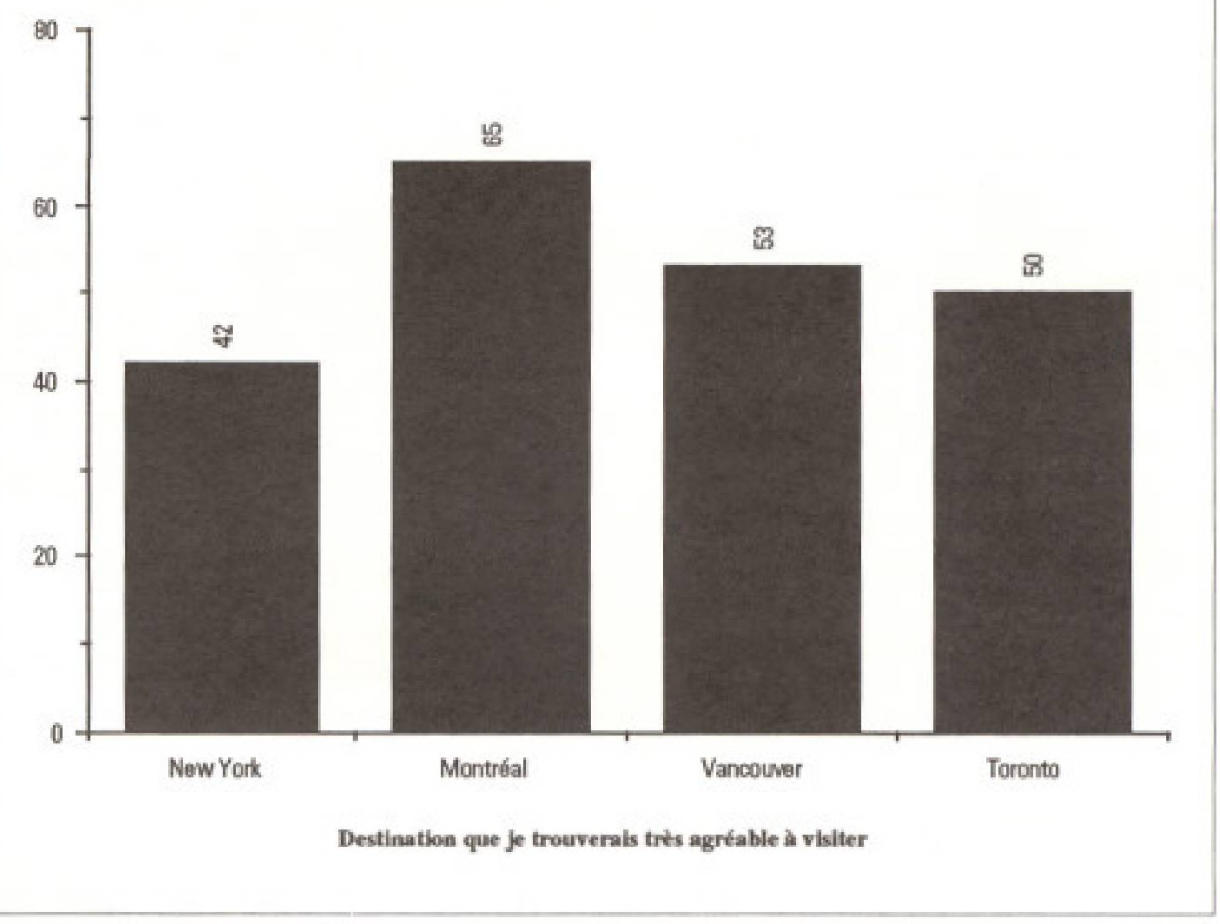

TABLEAU 6

L'image du Canada aux États-Unis 1985 par rapport à 1989

Lequel des deux pays l'emporte?
1. Destination agréable

2. Destination attirante

3. Habitants courtois

4. Cachet étranger

5. Paysages spectaculaires

6. Destinations populaires

7. Visites tourist./divertissements

8. Plein air

9. Retauration/ hébergement

10. Soleil / plage

11. Prix

12. Valeur
E.U.

É.U.

Canada

Canada

- Canada

Canada

Canada

- $\quad$ Canada

E..U.

É.U.

É.U.

É.U.

Canada

Canada

E.U.

É.U.

É.U

E.U

ne s'applique pas

Canada 
1985, sur les onze (11) points de comparaison établis entre les deux pays seulement, deux avantageaient le Canada alors qu'en 1989 surdouze points identifiés par Longwoods, sept (7) étaient en faveur du Canada, ce qui constitue un revirement plutôt spectaculaire. On constate aussi une amélioration au chapitre de la perception de la courtoisie des Canadiens, denos paysages spectaculaires et de la qualité de la restauration et de l'hébergement. Le seul point sur lequel les États-Unis ont fait un gain en terme de perception est lié au prix. Alors qu'en 1985, les Américains ne voyaient pas de différence notable entre les deux pays à ce chapitre, ils considèrent maintenant les États-Unis moins chers, ce quine peutqu'atténuer tous les gains obtenus àd'autreschapitres. Le Canadaet le Québec devront travaillerà changercette perception en mettant l'accent sur une meilleure connaissance denos réseaux d'hébergement à prix modique par exemple et en sensibilisant les intervenants touristiques à ce probleme si on ne veut pas que notre performance soit liée aux fluctuations du taux de change.

L'Enquête Longwoods a fait ressortir aussi la perception positive du Québec et de Montréal surtout en comparaison de l'Ontario et de Toronto. Cela nous indique qu'il existe des opportunités et un potentiel important aux États-Unis. II reste à trouver lafaçon laplus appropriee, avec des budgets relativement modestes, pour attirer les touristes américains chez nous. En effet, malgré la performance plus qu'honorable du Canada, le nombre de voyages d'agrément effectués par des Américains agés de 18 ans et plus au Canada n'a augmenté que de $3 \%$ entre 1985 et 1989 , passant de 7684000 a 7903000 . Même si l'Enquête Longwoods montre que le Canada était sur la bonne voie et que la campagne publicitaire fut efficace, il reste encore beaucoup de travail à faire pour matérialiser concrètement cette bonne perception et pour se distinguer des concurrents toujours plus nombreux et efficaces sur le marché américain. Cela ne pourra se faire qu'avec une meilleure connaissance du marché américain, une sélection de segments de marché judicieux et une concertation efficace de tous les intervenants touristiques du Québec intéresséspar cemarché. C'est uniquement à ce prix que l'on pourra accroitre de façon sensible le nombre de touristes américains.

$f$

NOTES

(1) EXTERNALAFFAIRS AND INTERNATIONAL TRADE ICANADA, Touriem Market Profiles - U.S. and Overseas, 1989.

(2) Voir entre autres SECOR, L'btude du comportoment et de l'attlitude des diffírents segments de elientbles tourietiques a Montról, 1989.

(3) Selon Laventhol \& Horwath, en 1989, le coût moyen d'une chambre d'hôtel à Montréal étaít le plus Glevé au Canada.

(4) Lire The Yankelovleh Monltor. Perspective on Leisure. Insighte Into The Leleure and Travel Markets annuel.

(5) Voir COUPET, Andre, Le towiame québécois a-i-it encove besoin de / $E$ tat?? in T6ores, vol. 9, no 1, mars 1990.

(6)O.C.T.G.Mv, Plan marketing 1990-1992, mai 1990. 